BULL. AUSTRAL. MATH. SOC.

P26E10

VOL. 33 (1986), 293-305.

\title{
ON A PROPERTY OF IDEALS OF
}

\section{DIFFERENTIABLE FUNCTIONS}

\author{
Oscar P. Bruno
}

Let $J \subseteq C^{\infty}\left(R^{n}\right)$ be any ideal. Since a function of the variables $\bar{t}=\left(t_{1}, \ldots, t_{n}\right)$ is a function of the variables $(\bar{t}, \bar{x})=\left(t_{1}, \ldots\right.$, $\left.t_{n}, x_{1}, \ldots, x_{p}\right)$ which does not depend on $\bar{x}$, we have $J \subseteq C^{\infty}\left(\mathbb{R}^{n+p}\right)$. of course, $J$ is not an ideal of $C^{\infty}\left(\mathbb{R}^{n+p}\right)$, but it generates an ideal that we call $J(\bar{t}, \bar{x})$. Consider the following statement (1) on $J:$ "Given any $f \in C^{\infty}\left(R^{n+p}\right), f \in J(\bar{t}, \bar{x})$ if and only if for every fixed $\bar{a} \in R^{p}, f(\bar{t}, \bar{a}) \in J^{\prime \prime}$.

In this paper we show that statement (1) holds for a large class of finitely generated ideals although not for all of them. We say that ideals satisfying statement (1) have line determined extensions. We characterize these ideals to be closed ideals $J(\bar{t})$ (in the sense of Whitney) such that for all $p \in \mathbb{N}$, the ideal $J(\bar{t}, \bar{x})$ is also closed. Finally, some non-trivial examples are developed.

Received 16 July 1985.

Copyright clearance Centre, Inc. Serial-fee code: 0004-9727/86 $\$ A 2.00+0.00$. 


\section{Introduction}

Let $J \subseteq C^{\infty}\left(\mathbb{R}^{n}\right)$ be any ideal. Since a function of the variables $\bar{t}=\left(t_{1}, \ldots, t_{n}\right)$ is a function of the variables $(\bar{t}, \bar{x})=\left(t_{1}, \ldots, t_{n}\right.$, $\left.x_{1}, \ldots, x_{p}\right)$ which does not depend on $\bar{x}$, we have $J \subseteq C^{\infty}\left(\mathbb{R}^{n+p}\right)$. of course, $J$ is not an ideal of $C^{\infty}\left(\pi R^{n+p}\right)$, but it generates an ideal that we call $J(\bar{t}, \bar{x})$. Consider the following statement (1) on $J$ : "Given any $f \in C^{\infty}\left(\mathbb{R}^{n+p}\right), f \in J(\bar{t}, \bar{x})$ if and only if for every fixed $\bar{a} \in \mathbb{R}^{p}$, $f(\bar{t}, \bar{a}) \in J^{\prime \prime}$. It is easily seen that statement (1) is, in general, false. Take for example $n=1$ and $J=\left\{h \in C^{\infty}(\mathbb{R}) \mid h\right.$ vanishes in a neighbourhood of $0 \in I R\}$ and let $f \in C^{\infty}\left(I^{2}\right)$ be a function which is null in $C=\{|x| \leq|y|\} \cup\{y=0\} \quad$ and different from zero everywhere else.

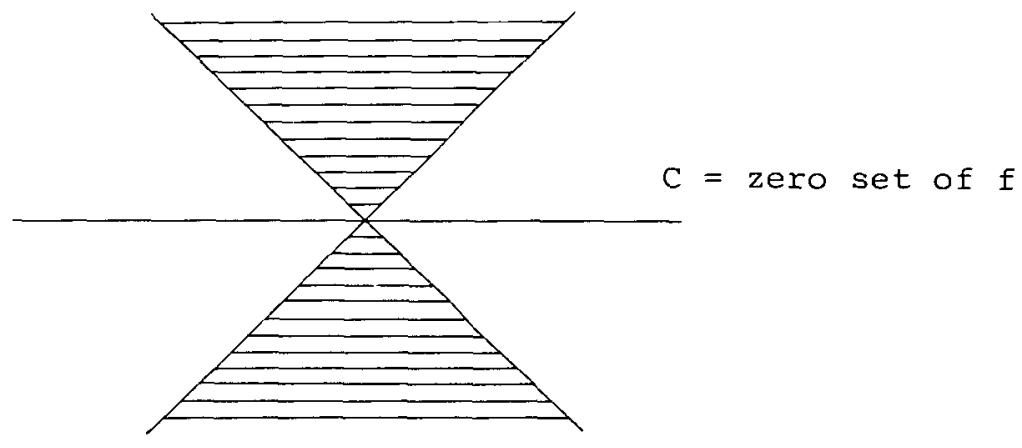

In this paper we show that statement (1) holds for a large class of finitely generated ideals (Corollary 11, Theorem 12) although not for all of them. We say that ideals satisfying statement (1) have line determined extensions. We characterize these ideals to be closed ideals (in the sense of Whitney [5]) such that for all $p \in \mathbb{N}$, the ideal $J(\bar{t}, \bar{x})$ is also closed (Theorem 10). Finally, some non trivial examples are developed.

\section{Ideals with line determined extensions}

Notation 1. i) $C^{\infty}\left(\mathbb{R}^{n}\right)$ will be understood as the ring of all 
$C^{\infty}$-mappings in the variables $\bar{t}=\left(t_{1}, \ldots, t_{n}\right)$ and $C^{\infty}\left(\mathbb{R}^{n+p}\right)$ as the ring of all $C^{\infty}$-mappings in the variables $\left(t_{1}, \ldots, t_{n}, x_{1}, \ldots, x_{p}\right)$.

ii) Let $J \subseteq C^{\infty}\left(\mathbb{R}^{n}\right)$ be any ideal. We will write $J(\bar{t})=J$ and $J(\bar{t}, \bar{x})=$ ideal generated by $J$ in $C^{\infty}\left(\mathbb{R}^{n+p}\right)$.

DEFINITION 2. An ideal $J \subseteq C^{\infty}\left(I^{n}\right)$ is said to have line determined extensions if and only if for every integer $p>0$ and function $f \in C^{\infty}\left(\mathbb{R}^{n+p}\right)$ the following condition holds: $f(\bar{t}, \bar{a}) \in J(\bar{t})$ for every fixed $\bar{a} \in \mathbb{R}^{p}$ implies $f \in J(\bar{t}, \bar{x})$.

(The reason for the name is that for $n=1, p=1$ the definition means: $f \in J(\bar{t}, \bar{x})$ if restricted to horizontal lines is in $J(\bar{t})$ ).

Remark 3. i) If $J=J(\bar{t})$ has line determined extensions so does any extension $J(\bar{t}, \bar{s})$. The converse also holds: if some extension of $J(\bar{t})$ has line determined extensions so does $J(\bar{t})$.

ii) Notice that the property on $J$ of having line determined extensions is an intrinsic property of the quotient $c^{\infty}$-ring. Explicitly, if $A=C^{\infty}\left(\mathbb{R}^{n}\right) / J$, then $J$ has line determined extensions if and only if for every $p \in \mathbb{I N}$ the family of all evaluations $e v_{x}: A \otimes_{\infty} C^{\infty}(I R p) \rightarrow A\left(x \in \mathbb{R}^{p}\right)$ is collectively monomorphic, or in other words, any "smooth polynomial" with coefficients in $A$ (that is, any element of $\left.A\left\{x_{1}, \ldots, x_{p}\right\}=A \otimes_{\infty} C^{\infty}\left(\mathbb{R}^{p}\right)\right)$ is nulI if it vanishes at every point of $\mathbb{R}^{p} \subseteq A^{p}$. (We have denoted here by $\otimes_{\infty}$ the $C^{\infty}$-tensor product of $c^{\infty}$-rings, see [1]).

DEFINITION 4. Recall that the $C^{\infty}$-compact open topology on $C^{\infty}\left(\mathbb{R}^{n}\right)\left(C^{\infty}-C O\right)$ is the topology for which a sequence $f_{k}$ of elements of $C^{\infty}\left(\mathbb{R}^{n}\right)$ converges to $f \in C^{\infty}\left(\mathbb{R}^{n}\right)$ if $f_{k}$ and all its derivatives converge uniformly on compact sets to $f$ and its respective derivatives. 
An example of an ideal which does not have line determined extensions is the ideal $J$ in the introduction. Notice that $J$ is not closed for the $C^{\infty}-C O$ topology. In fact we know that

PROPOSITION 5. If the ideal $J=J(t) \subseteq C^{\infty}\left(\mathbb{R}^{n}\right)$ has line determined extensions then it is closed for the $c^{\infty}-c 0$ topology (and by 3 , so is any extension $J(\bar{t}, \bar{x})$ of $i t)$,

Proof. Assume that there exists a sequence $f_{k}, k \in \mathbb{N}, f_{k} \in J$ $C^{\infty}$-CO converging to $f \in C^{\infty}\left(\mathbb{R}^{n}\right)$. By 6 , below, we may assume (taking a subsequence) that there exists $H \in C^{\infty}\left(\mathbb{R}^{n+1}\right)$ such that $H(\bar{t}, 1 / k)=$ $H(\bar{t},-1 / k)=f_{k}(\bar{t}), H(\bar{t}, 0)=f(\bar{t})$ and $\frac{\partial H}{\partial s}(\bar{t}, s) \in J(\bar{t})$ for any fixed $s \in \mathbb{R}$. Now, $H(\bar{t}, 1)-H(\bar{t}, 0)=\int_{0}^{1} \frac{\partial H}{\partial s}(\bar{t}, s) d s$. Since $\frac{\partial H}{\partial s}(\bar{t}, s) \in J(\bar{t})$ for every fixed $s \in \mathbb{R}$ and $J(\bar{t})$ has line determined extensions, we have that $\frac{\partial H}{\partial s}(\bar{t}, s) \in J(\bar{t}, s)$, or in other words we have:

$$
\frac{\partial H}{\partial s}(\bar{t}, s)=\sum_{i=1}^{r} A_{i}(\bar{t}, s) h_{i}(\bar{t})
$$

with $A_{i} \in C^{\infty}\left(\mathbb{R}^{n+1}\right), h_{i} \in J$. It follows that $H(\bar{t}, 1)-H(\bar{t}, 0)=$ $\sum_{i=1}^{r} h_{i}(\bar{t}) \int_{0}^{1} A_{i}(\bar{t}, s) d s$ and this says that $H(\bar{t}, 1)-h(\bar{t}, 0) \in J(\bar{t})$. But $H(\bar{t}, 1) \in J(\bar{t})$, so we actually have $f(\bar{t})=H(\bar{t}, 0) \in J$.

LEMMA 6. Let $J=J(\bar{t})$ be any ideal, $J \subseteq C\left(\mathbb{R}^{n}\right)$, and let $f_{k} \in J$ be a sequence $C^{\infty}-c 0$ converging to $f \in C^{\infty}\left(\mathbb{R}^{n}\right)$. Then, there exists a subsequence $f_{k_{\ell}}$ and $H \in C^{\infty}\left(\mathbb{I R}^{n+1}\right)$ such that 


$$
\left\{\begin{array}{l}
H(\bar{t}, 1 / \ell)=H(\bar{t},-1 / l)=f_{k_{\ell}}(\bar{t}) \\
H(\bar{t}, 0)=f(\bar{t}) \\
\frac{\partial H}{\partial s}(\bar{t}, s) \quad J(\bar{t}) \text { for every fixed } s \in R .
\end{array}\right.
$$

Proof. Let $K_{\ell}=[-\ell, \ell]^{n}$ be the closed $n$-cube. By hypothesis, there exists $k_{\ell}$ such that $\left|D^{a}\left(f_{k_{\ell}}-f\right)\right|<e^{-\ell}$ in $k_{\ell}$ for every $a$ such that $|a|=\sum a_{i} \leq \ell$. Now take $\phi \in C^{\infty}(\mathbb{R})$ such that $\phi([-1 / 2,1 / 2])=1$ and $\operatorname{supp}(\phi) \subseteq(-1,1)$, and write $\phi_{\ell}(s)=$ $\phi(2 \ell(\ell+1)(s-1 / \ell))$. We have $\operatorname{supp}\left(\phi_{\ell}\right) \subseteq(1 / \ell-1 / 2 \ell(\ell+1), 1 / \ell+$ $1 / 2 \ell(\ell+1))$ and $\Phi_{\ell}([1 / \ell-1 / 4 \ell(\ell+1), 1 / \ell+1 / 4 \ell(\ell+1)])=1$. Notice that $\operatorname{supp}\left(\phi_{\ell}\right) \cap \operatorname{supp}\left(\phi_{k}\right)=\emptyset$ if $\ell \neq k$. Let us define

$$
H(\bar{t}, s)=\left\{\begin{array}{l}
\phi_{\ell}(s) \cdot f_{k_{\ell}}(\bar{t})+\left(1-\phi_{\ell}(s)\right) f_{k_{\ell+1}}(\bar{t}) \text { if } \\
1 /(\ell+1) s s \leq 1 / \ell \text { or }-1 / \ell \leq s \leq-1 /(\ell+1) \\
f_{k_{1}}(\bar{t}) \text { if }|s|>1 \\
f(\bar{t}) \text { if } s=0 .
\end{array}\right.
$$

One may easily verify that $H$ is $C^{\infty}$ and that $\frac{\partial H}{\partial s}(\bar{t}, 0)=0 \in J$. The fact that $\frac{\partial H}{\partial s} \in J(\bar{t})$ for every fixed $s \neq 0$ is immediate.

DEFINITION 7. An ideal $J \subseteq C^{\infty}\left(\mathbb{R}^{n}\right)$ is said to be universally closed if and only if $J$ itself and any extension $J(\bar{t}, \bar{x})$ of $J$ are closed.

We remark that 5 says that ideals which have line determined extensions are universally closed. E. Dubuc pointed out that a derivativeintegral argument like that of 5 should allow us to use Riemann sums to prove its converse. Actually we can use Riemann sums directly.

PROPOSITION 8. Let $J \subseteq C^{\infty}\left(\mathbb{R}^{n}\right)$ be cony ideal, and let $f(\bar{t}, s) \epsilon$ 
$C^{\infty}\left(\mathbb{R}^{n+1}\right)$ be such that $f\left(\bar{t}, s_{0}\right) \in J(\bar{t})$ for every fixed $s_{0} \in \mathbb{R}$. Then $f(\bar{t}, s)$ is in the $C^{\infty}-C O$ closure of $J(\bar{t}, s)$.

In order to prove this, we need lemma 9, whose standard proof we omit.

LEMMA 9. Let $f \in C^{\infty}\left(\mathbb{R}^{n+1}\right)$. Then we have

i) the Riemonn sums $S_{n}^{\alpha}(\bar{t})=\sum_{i=-n}^{n} f\left(\bar{t}, \frac{a \cdot i}{n}\right) \cdot \frac{a}{n} \quad c^{\infty}-C 0$ converge to $s^{a}(\bar{t})=\int_{-\alpha}^{a} f(\bar{t}, w) d w$ as $n \rightarrow \infty$,

ii) let $\rho \in C^{\infty}(\mathbb{R})$ be the function

$$
\rho(s)=\left\{\begin{array}{l}
\exp \left(-1 /\left(1-s^{2}\right) \quad \text { if }|s|<1\right. \\
0 \quad \text { otherwise, }
\end{array}\right. \text { and }
$$

$\rho_{j}$ be the kernel $\rho_{j}(s)=\frac{j \cdot \rho(j s)}{f \rho(s) d s}$; then the sequence $f_{j}^{a}(\bar{t}, s)=$ $\int_{-a}^{a} f(\bar{t}, w) \rho_{j}(s-w) d w \quad c^{\infty}-c o$ converges to $f(\bar{t}, s)$ as $n, a \rightarrow \infty$.

Proof of 8 . Let $f \in C^{\infty}\left(\mathbb{R}^{n+1}\right)$ be such that for every fixed $s_{0} \in \mathbb{R}$, $f\left(\bar{t}, s_{0}\right) \in J(\bar{t})$. We must show that $f(\bar{t}, s) \in \overline{J(\bar{t}, s)}=C^{\infty}-C O$ closure of $J(t, s)$.

We have that, for every fixed $w \in R f(\bar{t}, w) \cdot \rho_{j}(s-w) \in J(\bar{t}, s)$

(see 9.ii) for the definition of $\rho_{j}$ ). So, it follows from 9.i) that $\int_{-a}^{a} f(\bar{t}, w) j(s-w) d w \in \overline{J(\bar{t}, s)}$.

Then, from 9.ii) we deduce that $f(\bar{t}, s) \in \overline{J(\bar{t}, s)}$. The following theorem follows immediately from 5 and 8 .

THEOREM 10. An ideal $J \subseteq C^{\infty}\left(\mathbb{R}^{n}\right)$ has line determined extensions if and only if it is universally closed. 
It is known (see [2]) that ideals generated by a finite number of analytic functions are closed, and thus universally closed. We have:

COROLLARY 11 . Let $f_{1}, \ldots, f_{r}$ be analytic functions in $C^{\infty}\left(\mathbb{I R}^{n}\right)$. Then the ideal generated by $f_{1}, \ldots, f_{p}$ has line determined extensions. This corollary gives us a large class of finitely generated ideals having line determined extensions. However not all finitely generated ideals are such. It suffices to recall that finitely generated ideals are not necessarily closed. Explicitly, take $J=\left(e^{-1} / t^{2}\right) \subseteq C^{\infty}(\mathbb{R})$ which is

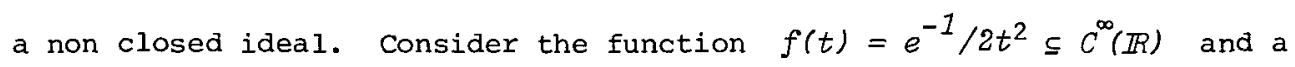
sequence of functions $f_{k} \in C^{\infty}(\mathbb{R}) \quad C^{\infty}-C O$ converging to $f$ and such that $f_{k}$ vanishes in a neighbourhood of $0 \in \mathbb{R}$. If we now apply 6 to $f_{k}$ and $f$ we obtain a function $H \in C^{\infty}\left(\mathbb{R}^{2}\right)$ such that $\frac{\partial H}{\partial s}(\bar{t}, s) \in J$ for every fixed $s \in \mathbb{R}$ and $H(t, 0)=e^{-1} / 2 t^{2}$. In other words, the function $F=\frac{\partial H}{\partial s}$ is such that for any fixed $s \in \mathbb{R}$ there exists $h_{s}(t) \in C^{\infty}(\mathbb{R})$ such that $F(t, s)=h_{s}(t) e^{-1} / t^{2}$. But $h_{s}(t)$ cannot be a $C^{\infty}$-function of both variables $t$ and $s$. If it were, it would follow as in 5 that $H(t, 0)=e^{-1 / 2 t^{2}} \in J$ which is clearly impossible.

THEOREM 12. Let $J \subseteq C^{\infty}\left(\mathbb{R}^{n}\right)$ be a finitely generated ideal with line determined extensions and consider an $n$-tuple $\bar{g}=\left(g_{1}, \ldots, g_{n}\right)$ of elements of $C^{\infty}\left(\mathbb{R}^{l}\right)$, that $i s, \bar{g}: \mathbb{R}^{l} \rightarrow \mathbb{R}^{n}$. Assume that $g_{1}, \ldots, g_{n}$ are independent (that is the jacobion of $\bar{g}$ has rank $n$ ) at every point of $\vec{g}^{-1}(Z(J))$. Then, the ideal $J_{g}=$ ideal generated in $C^{\infty}\left(\mathbb{R}^{2}\right)$ by $\{h \circ \vec{g} \mid h \in J\}$ has line determined extensions.

Proof. Take $f \in C^{\infty} a R^{l+p}$, such that for every fixed $\bar{a} \in \mathbb{I}^{p}$, $f(\bar{s}, \bar{a}) \in J_{g}$. We must show that $f(\bar{s}, \bar{x}) \in J_{g}(\bar{s}, \bar{x}) \quad\left(\bar{s}\right.$ ranges over $\mathbb{I R}^{\ell}$ and $\vec{x}$ over $\mathbb{I R}^{p}$ ). Since $J_{g}$ is of local character (because it is 
finitely generated, see [1]) it suffices to show that for every $\left(\bar{s}_{0}, \bar{x}_{0}\right) \in \mathbb{R}^{l+p}$ there exists an open neighbourhood $V$ of $\left(\bar{s}_{0}, \bar{x}_{0}\right)$ such that $\left.f\right|_{V}$ is in the ideal $\left.J_{g}(\bar{s}, \bar{x})\right|_{V}$ of $C(V)$. (If $I \subseteq C^{\infty}(\mathbb{R})^{n}$ ) is any ideal then $I_{V}$ is the ideal generated in $C(V)$ by $\left.\left\{\left.h\right|_{V} \mid h \in I\right\}\right)$.

The latter is trivial if $\bar{s}_{0} \notin Z\left(J J_{g}\right)$. So take $\left(\bar{s}_{0}, \bar{x}_{0}\right)$ with $\bar{s}_{0} \in Z\left(J_{g}\right)=\bar{g}^{-1}(Z(J))$. Denote by $\bar{s}_{0}^{\prime \prime} \in I R^{l-n}$ the last $\ell-n$ coordinates of $\bar{s}_{0}$. Since by hypothesis $g_{1}, \ldots, g_{n}$ are independent at $s_{0}$, by the inverse function theorem we may assume (changing the order of the variables if necessary) that there exist open neighbourhoods $U^{\prime}$ of $\bar{s}_{O} \in \mathbb{R}^{\ell}, W^{\prime}$ of $\left(\bar{g}\left(\bar{s}_{0}^{)}, \bar{s}_{0}^{\prime \prime}\right) \in \mathbb{R}^{l}\right.$ and a diffeomorphism $\bar{\psi}: W^{\prime} \rightarrow U^{\prime}$ such that $\bar{g} \circ \bar{\psi}: W^{\prime} \rightarrow \mathbb{R}^{n}$ equals the projection $\bar{\pi}$ on the first $n$ coordinates. We know that $f(\bar{s}, \bar{a}) \in J_{g}$ for every fixed $\bar{a} \in \mathbb{R}^{p}$, that is, there exist functions $\left.A_{i}^{\bar{\alpha}} \in C^{\infty}(\mathbb{R})^{\ell}\right)^{\bar{g}}$ such that $f(\bar{s}, \bar{a})=$ $\sum_{i=1}^{n} A_{i}^{\bar{a}} h_{i}(\bar{g})\left(a \in \mathbb{R}^{p}\right)$, where $h_{1}, \ldots, h_{p}$ are generators of $J$. It follows that, in $W^{\prime}, f(\bar{\psi}, \bar{a})=\sum_{i=1}^{r} A_{i}^{\bar{a}}(\psi) . h_{0} \cdot \bar{\pi} \cdot$ Setting $\bar{s}^{\prime}=$ $\left(s_{1}, \ldots, s_{n}\right), \bar{s}^{\prime \prime}=\left(s_{n+1}, \ldots, s_{\ell}\right)$, we have equivalently $f\left(\bar{\psi}\left(\bar{s}^{\prime}, \bar{s}^{\prime \prime}\right), \bar{a}\right)=$ $\sum_{i=1}^{r} A_{i}^{\bar{a}}\left(\psi\left(\bar{s}^{\prime}, \bar{s}^{\prime \prime}\right) h_{i}\left(\bar{s}^{\prime}\right)\right.$. Now take an open neighbourhood $W$ of $\left(g\left(\bar{s}_{0}\right), \bar{s}_{0}^{\prime \prime}\right)$ such that $\bar{W} \subseteq W^{\prime}$ and a function $\phi \in C^{\infty}\left(\mathbb{R}^{l}\right)$ such that $Z(\phi)=\mathbb{R}^{\ell} \backslash W$. We have that $H(\bar{s}, \bar{x})=\phi(\bar{s}) \cdot f(\psi(\bar{s}), \bar{x})$ and $\phi(\bar{s}) A_{i}^{a}(\bar{\psi}(\bar{s}))$ are defined and $C^{\infty}$ in all of $\mathbb{R}^{\ell+p}$ and $\mathbb{R}^{\ell}$ respectively. Moreover we have $H(\bar{s}, \bar{a})=\sum_{i=1}^{r} \phi(\bar{s}) A_{i}^{a}(\bar{\psi}(\bar{s})) \cdot h_{i}(\bar{s})$. So, for every fixed $\left(\bar{s}^{\prime \prime}, \bar{a}\right)$ $\epsilon \mathbb{R}^{(\ell-n)+p}, H\left(\bar{s}^{\prime}, \bar{s}^{\prime \prime}, \bar{a}\right) \in J\left(\bar{s}^{\prime}\right)$. It follows that $H\left(\bar{s}^{\prime}, \bar{s}^{\prime \prime}, \bar{x}\right) \in J\left(\bar{s}^{\prime}, \bar{s}^{\prime \prime}, \bar{x}\right)$, 
since $J$ has line determined extensions. And, since $\phi$ does not vanish in $W,\left.\left.f(\bar{\psi}, \bar{x})\right|_{W \times \mathbb{R}^{p}} \in J\left(\bar{s}^{\prime}, \bar{s}^{\prime \prime}, \bar{x}\right)\right|_{W \times \mathbb{R}^{p}}$. Then, letting $U=$ $\bar{\psi}(W),\left.\left.f(\bar{s}, \bar{x})\right|_{U \times \mathbb{R}^{p}} \bar{J}_{\bar{g}}(\bar{s}, \bar{x})\right|_{U \times \mathbb{R}^{p}}$ and so, $V=U \times \mathbb{R}^{p}$ is the required neighbourhood of $\left(\bar{s}_{0}, \bar{x}_{0}\right)$.

\section{Examples of ideals with line determined extensions}

EXAMPLE 1. All those ideals obtained by a suitable combination of 11 and 12.

i) Ideals generated by a finite number of independent functions (meaning independent in the set of their common zeroes). An instance of this is the ideal $J=\left(f(\bar{t}) t_{n+1}-1\right) \subseteq C^{\infty}\left(\pi R^{n+1}\right)$, where $f \in C^{\infty}\left(\mathbb{R}^{n}\right)$ is a function such that $Z(f)=\mathbb{I}^{n} \backslash U$ for a certain open subset $U$ of $\mathbb{I R}^{n}$. This ideal $J$ is the ideal of presentation of the ring $C^{\infty}(U)$ as a quotient of $C^{\infty}\left(\mathbb{R}^{n+1}\right)$ (see [1]). Of course $\left(f(\bar{t}) \cdot t_{n+1}-1\right)$ is a nonanalytic function.

ii) The ideal of presentation of any weil algebra (see [4]) as a quotient of $C^{\infty}\left(\mathbb{R}^{n}\right)$ for some $n \in \mathbb{N}$. We have here the simple example $\left(x^{2}\right) \subseteq C^{\infty}(\mathbb{R})$. It may be seen directly (Taylor series expansion) that this ideal has line determined extensions.

Remark 13. From 10,11 and 12 it follows that not only ideals generated by a finite number of analytic functions are closed. Actually if $h_{1}, \ldots, h_{r} \in C^{\infty}\left(R^{n}\right)$ are analytic functions and $g_{1}, \ldots, g_{n} \in C^{\infty}\left(\mathbb{R} R^{\ell}\right)$ are independent in $\bar{g}^{-1}\left(2\left(h_{1}, \ldots, h_{r}\right)\right)$ then $h_{1}\left(g_{1}, \ldots, g_{n}\right), \ldots$, $h_{p}\left(g_{1}, \ldots, g_{n}\right)$ generate a closed ideal. We consider now some examples of ideals with line determined extensions which fall outside the context above. 
EXAMPLE 2. It follows from a result of A. P. Calderon (unpublished) that the ideal of functions flat at $t_{0} \in I R$ is universally closed (recall that "flat at $t_{0}$ " means "function and all derivatives vanish at $t_{0}$ ). Van que and Reyes ([3]) generalised this result and showed that the ideal of flat functions on a closed set is universally closed (and thus has line determined extensions).

THEOREM 14. (CaZderon - Reyes - van Quê, see [3])

Let $X$ and $y$ be closed subsets of $\mathbb{R}^{n}$ and $\mathbb{R}^{p}$ respectively. Let $J=J(\bar{t}) \subset C^{\infty}\left(\mathbb{R}^{n}\right), I=I(\bar{x}) \subset C^{\infty}\left(\mathbb{R}^{p}\right)$ and $K=K(\bar{t}, \bar{x})$ be the ideals of flat functions on $X, Y$ and $X \times Y$ respectively. Then, $K(\bar{t}, \bar{x})=J(\bar{t}, \bar{x})+I(\bar{x}, \bar{t})$.

clearly, considering $Y=\mathbb{R}^{p}$ it follows that $J$ has line determined extensions.

EXAMPLE 3. The ideal $J$ of all functions $h \in C^{\infty}(I R)$ such that $h(1 / n)=0$ for every $n \in \mathbb{I N}$. In order to see that $J$ has line determined extensions, take $p \in \mathbb{I N}$ and a function $f \in C^{\infty}\left(R^{1+p}\right)$ vanishing on $\left\{\frac{I}{n} \mid n \in \mathbb{N}\right\} \times \mathbb{R}^{p}$. We prove two lemmas.

LEMMA 15. Let $k \in \mathbb{I N}$. Assume that $h(t, \bar{x}) \in C^{\infty}\left(I R^{1+p}\right)$ is such that $\frac{\partial^{j} h}{\partial t^{j}}(1 / n, \bar{x})=0$ for every $\bar{x} \in I R^{p}, n \in \mathbb{I N}$ and $0 \leq j \leq k$. Let $U=\left\{(t, \bar{x}) \in \mathbb{R}^{1+p} \mid t>0\right.$ and $\left.t \neq 1 / n, n \in \mathbb{I V}\right\}$ and $g(t, \bar{x})=$ $\frac{h(t, \bar{x})}{\sin ^{k}(\pi / t)}: U \rightarrow R$. Then for any $\bar{x}_{0} \in \mathbb{I R}^{p}$ we have

$$
\begin{aligned}
& \lim _{(t, \bar{x}) \rightarrow\left(0, \bar{x}_{0}\right)} g(t, \bar{x})=0 . \\
& (t, \bar{x}) \in U
\end{aligned}
$$

Proof. We prove this by induction. The assertion above is trivial if $k=0$. So take $k>0$, assume the assertion holds for $k-1$ but there exists $h \in C^{\infty}\left(\mathbb{R}^{1+p}\right)$ which for $\bar{x} \in \mathbb{R}^{p}$ fixed has a 
a zero of order $k$ at $1 / n, n \in \mathbb{N}$ such that the limit above either does not exist or is different from zero.

In this case, there exists a sequence $\left(t_{\ell}, \bar{x}_{\ell}\right)$ converging to $\left(0, \bar{x}_{0}\right), t_{\ell}>0, t_{\ell} \neq 1 / n, n \in \mathbb{N}$ such that

$$
\lim _{\ell \rightarrow \infty} \frac{h\left(t_{\ell}, \bar{x}_{\ell}\right)}{\sin ^{k}\left(\pi / t_{\ell}\right)}=a
$$

with $a \in \mathbb{R}, \quad a \neq 0$ or $a=\infty$. Taking a subsequence we may assume that $\sin \left(\pi / t_{\ell}\right)$ is a convergent sequence. Notice that it necessarily must approach zero, and so, $\left|\cos \pi / t_{\ell}\right|$ must approach 1 . Let $n_{\ell} \in \mathbb{N}$ be such that $n_{\ell} \pi$ realises the distance between $\pi / t_{\ell}$ and the set $\{n \pi: n \in \mathbb{N}\}$. We have that

$$
\frac{h\left(t_{\ell}, \bar{x}_{\ell}\right)}{\sin ^{k}\left(\pi / t_{\ell}\right)}=\frac{h\left(t_{\ell}, \bar{x}_{\ell}\right)-h\left(1 / n_{\ell}, \bar{x}_{\ell}\right)}{\sin ^{k}\left(\pi / t_{\ell}\right)-\sin ^{k}\left(\pi / 1 /_{n_{\ell}}\right)}
$$

and, by the Cauchy mean value theorem this equals

$$
\frac{-\xi_{\ell}^{2}}{\pi} \cdot \frac{\frac{\partial h}{\partial t}\left(\xi_{\ell}, \bar{x}_{\ell}\right)}{k \sin ^{k-1}\left(\pi / \xi_{\ell}\right) \cdot \cos \pi / \xi_{\ell}}
$$

for some $\bar{\xi}_{\ell}$ between $t_{\ell}$ and $1 / n_{\ell}$. Now, by the inductive hypothesis,

$$
\frac{\frac{\partial h}{\partial t}\left(\xi_{\ell}, \bar{x}_{\ell}\right)}{\sin ^{k-1}\left(\pi / \xi_{\ell}\right)} \text { tends to zero }
$$

and $\left|\cos \left(\pi / \xi_{\ell}\right)\right|$ tends to 1 .

LEMMA 16. Let $f(t, \bar{x}) \in C^{\infty}\left(\mathbb{R}^{1+p}\right)$ be such that $f(1 / n, \bar{x})=0$. Notice that, since $\sin (\pi / t)$ has a zero of order one at $t=1 / n$, the function $f(t, \bar{x}) / \sin (\pi / t): U \rightarrow \mathbb{R}$ may be extended to a $C^{\infty}$ map $g: \mathbb{R}>0 \times \mathbb{R}^{p} \rightarrow \mathbb{R}$. Then, the function 


$$
\tilde{g}(t, \bar{x})=\left\{\begin{array}{lll}
g(t, x) & \text { if } & t>0 \\
f(t, x) & \text { if } & t \leq 0
\end{array}\right.
$$

is in $C^{\infty}\left(\pi R^{1+p}\right.$, and it is flat on $\{0\} \times I^{p}$.

Proof. We need only show that $g$ and all its derivatives tend to zero as $(t, \bar{x})$ tend to $\left(0, \bar{x}_{0}\right)$ for any $\bar{x}_{0} \in \mathbb{R}^{p}$.

i) That $g$ tends to zero follows from 15 .

ii) $g_{t}$ tends to zero: in $U, g_{t}$ equals

$$
\frac{\frac{\partial f}{\partial t}(t, \bar{x}) \sin (\pi / t)+\pi / t^{2} \cos (\pi / t) \cdot f(t, \bar{x})}{\sin ^{2}(\pi / t)} .
$$

The numerator is in $C^{\infty}\left(I R^{1+p}\right.$ ) (notice that since $f$ vanishes at $t=1 / n, n \in \mathbb{N}$, it follows that $f$ is flat on $\{0\} \times \mathbb{I}^{p}$, and so, $\left.\frac{f(t, \bar{x})}{t^{2}} \cdot \cos (\pi / t) \quad C^{\infty}\left(\mathbb{R}^{7+p}\right)\right)$ and the quotient is $C^{\infty}$ in a neighbourhood of $1 / n$. It follows that for fixed $\bar{x} \in \mathbb{R}^{p}$ the numerator has a zero of order not less than two at $\frac{1}{n}, n \in \mathbb{N}$. Now we may apply 15.

iii) Any other derivative of $g$ is treated in a similar way. Now we return to Example 3). Consider the functions

$$
A(t)=\left\{\begin{array}{ccc}
\sin (\pi / t) & \text { if } t>0 \\
1 & \text { if } t \leq 0
\end{array}\right.
$$

and $\tilde{g}$ as defined in 16 . We have $A \cdot \tilde{g}=f$. Since $g$ is flat on $\{0\} \times \mathbb{I R}^{p}$, it follows from Example 2 that there exists a function $\phi \in C^{\infty}(\mathbb{R})$ flat at zero which divides $\tilde{g}$, that is, there exists $h \in C^{\infty}\left(\mathbb{R}^{1+p}\right)$ such that $\tilde{g}(t, \bar{x})=\phi(t) \cdot h(t, \bar{x})$. So, $f(t, \bar{x})=$ $(A(t), \phi(t)) \cdot h(t, \bar{x})$ and it may be easily seen that $(A(t) \cdot \phi(t))$ is a $C^{\infty}$ function and so, as it vanishes at $t=1 / n$, it is in $J$. 
Remark 17. The constructions in this example can be generalised to treat the ideal $K$ whose functions vanish at $1 / n$ with an order $o_{n} \in \mathbb{N} \cup\{\infty\}$ provided that the finite orders are bounded.

Reyes and van Quê ([3]) raise the question of whether every closed ideal is universally closed. We believe that this is true for dimension one:

Conjecture. Every closed ideal $J \subseteq C^{\infty}(\mathbb{R})$ is universally closed.

\section{References}

[1] E. Dubuc, "C $C^{\infty}$-Schemes", Amer. J. Math. 103 (1981) 683-690.

[2] B. Malgrange, Ideals of Differentiable Functions, (Oup, 1966).

[3] G. E. Reyes and N. Van Quê, "Smooth functors and synthetic calculus", The L.E.J. Brouwer Centenary Symposium, A. S. Troelstra and D. van Dalen (eds.), (North Holland 1982), 377-395.

[4] A. Weil, "Theorie des points proches sur les varietes differetiables", Geometrie differentielle. (Colloques Internationaux du Centre National de la Recherche Scientifique, Strasbourt, 1953. Centre National de la Recherche Scientifique, Paris, 1953), 111-117.

[5] H. Whitney, "On ideals of differentiable functions", Amer. J. Math. 70 (1948), 635-658.

Cuidad Universitaria

Pab.no. 1, Depto de Mathematics

1428 - BUENOS AIRES

Republica Argentina. 